\title{
Urinary steroid profiling in diagnostic evaluation of an unusual adrenal mass
}

\author{
N F Lenders $1,2,3$ and J R Greenfield $1,2,3$ \\ 1Diabetes and Metabolism, Garvan Institute of Medical Research, Sydney, New South Wales, Australia, ${ }^{2}$ Department \\ of Endocrinology, St Vincent's Hospital, Sydney, New South Wales, Australia, and ${ }^{3}$ St Vincent's Clinical School, \\ University of New South Wales, Sydney, New South Wales, Australia
}

Correspondence should be addressed to N F Lenders

Email

n.lenders@garvan.org.au

\section{Summary}

Adrenal oncocytomas are rare tumours, with only approximately 160 cases reported in the literature. We report the use of urinary steroid profiling as part of their diagnostic evaluation and prognostication. A 45-year-old woman presented with clinical features of hyperandrogenism. Serum biochemistry confirmed androgen excess and computed tomography (CT) demonstrated a $3.2 \mathrm{~cm}$ adrenal tumour with density $39 \mathrm{HU}$ pre-contrast. Urine steroid profiling showed elevated tetrahydro-11 deoxycortisol (THS), which is associated with adrenal malignancy. Laparoscopic adrenalectomy was performed, and histopathology diagnosed adrenal oncocytoma. Serum and urinary biochemistry resolved post-operatively and remained normal at 1-year follow-up.

\section{Learning points:}

- Differential diagnosis of adrenal masses is challenging. Current techniques for differentiating between tumour types lack sensitivity and specificity.

- 24-h urinary steroid profiling is a useful tool for reflecting steroid output from adrenal glands. Gas chromatography-mass spectrometry (GC-MS) of urinary steroid metabolites has sensitivity and specificity of $90 \%$ for diagnosing adrenocortical carcinoma.

- Adrenal oncocytoma are rare tumours. Differentiating between benign and malignant types is difficult. Data guiding prognostication and management are sparse.

\section{Background}

Adrenal tumours are a common incidental finding on abdominal imaging. Prevalence is approximately 5\% in the general population and increases with age (1). Adrenal oncocytic tumours comprise a rare subset of these, with only approximately 160 cases reported in the literature (2).

Differentiating between adrenal tumour types poses a significant diagnostic challenge. Serum biochemistry determines the presence of adrenal hormone excess. Distinguishing between benign and malignant tumours is more difficult, because of limited accuracy of diagnostic tools. Urinary steroid profiling offers a promising adjunct to current techniques for distinguishing between tumour types (1).

Herein, we present a patient with adrenal oncocytoma. To our knowledge, this is the first reported case of adrenal oncocytoma investigated with urinary steroid profiling.

\section{Case presentation}

A 45-year-old woman was referred in January 2018 for investigation and management of amenorrhoea, acne and hirsutism following removal of her contraceptive etonogestrel implant, 8 months prior to presentation. 
She denied features of hypercortisolism; specifically, there was no skin thinning, easy bruising or hypertension. There was no history of flushing or palpitations. Menarche was reached at age 13 with regular menses until the contraceptive etonogestrel implant was placed in 2014. Her symptoms developed following its removal in April 2017.

Other background was significant only for a history of histiocytic necrotizing lymphadenitis diagnosed in 1999. Her only medications were intermittent vitamin D and calcium. There were no drug allergies. Socially, she lived alone, was single and had not had children. She worked as chef. She did not smoke and drank alcohol socially. There was no relevant family history.

On examination, she was obese with a weight of $86.9 \mathrm{~kg}$ and BMI of $30.1 \mathrm{~kg} / \mathrm{m}^{2}$. There were no signs of Cushing's syndrome. Blood pressure was 130/70 mmHg. Cardiovascular, respiratory, thyroid and abdominal examinations were unremarkable.

\section{Investigation}

Initial investigations demonstrated normal full blood count, renal function and liver function. Serum hormonal studies (immunoassay) are summarised in Table 1. Testosterone and androstenedione were elevated, with normal SHBG. DHEAS andoestradiol were normal with gonadotropins in mid normal range. Baseline $17 \mathrm{OH}$ progesterone was elevated, albeit with no significant stimulation with ACTH. Cortisol did not suppress on $1 \mathrm{mg}$

Table 1 Initial serum laboratory data.

\begin{tabular}{|c|c|c|}
\hline Test & Value & Reference range \\
\hline FSH & 6.4 & 1.7-13.0 IU/L \\
\hline $\mathrm{LH}$ & 14.4 & 1.0-13.0 IU/L \\
\hline Oestradiol & 891 & 50-1000 pmol/L \\
\hline Prolactin & 236 & 50-500 mIU/L \\
\hline ACTH & $<1.5$ & 0.0-12.0 pmol/L \\
\hline Cortisol (1 mg DST) & 132 & $<50 \mathrm{nmol} / \mathrm{L}$ \\
\hline Testosterone & 5.0 & 0-1.8 nmol/L \\
\hline $\mathrm{SHBG}$ & 58 & 20-110 nmol/L \\
\hline $170 \mathrm{H}$ Progesterone & 15.8 & $1.0-6.5 \mathrm{nmol} / \mathrm{L}$ \\
\hline Androstenedione & 28 & $2.2-10.5 \mathrm{nmol} / \mathrm{L}$ \\
\hline DHEAS & 4.7 & 2.2-9.1 $\mu \mathrm{mol} / \mathrm{L}$ \\
\hline Aldosterone & 202 & 60-980 pmol/L \\
\hline Renin activity & $<90$ & $130-2350 \mathrm{fmol} / \mathrm{L} / \mathrm{s}$ \\
\hline
\end{tabular}

$17 \mathrm{OH}$ Progesterone, 17 hydroxy-progesterone; ACTH, adrenocorticotropic hormone; DHEAS, DHEA sulphate; DST, dexamethasone suppression test; $\mathrm{FSH}$, follicle-stimulating hormone; fT4, free thyroxine; $\mathrm{GH}$, growth hormone; IGF-1, insulin-like growth factor 1; LH, luteinising hormone; SHBG, sex hormone-binding globulin; $\mathrm{TSH}$, thyroid-stimulating hormone. Abnormal values are in bold face. overnight dexamethasone suppression test. Computed tomography (CT) adrenals demonstrated a $3.2 \mathrm{~cm}$ left adrenal tumour with density measurements of $39 \mathrm{HU}$ precontrast and washout of $65 \%$ (Fig. 1). 24-h urine steroid metabolites (gas chromatography-mass spectrometry) are demonstrated in Table 2. Marked elevation of tetrahydro-11 deoxycortisol (THS) was noted, which is associated with adrenocortical carcinoma.

\section{Treatment}

Left laparoscopic adrenelectomy was performed, with perioperative glucocorticoid cover. Histopathology confirmed adrenal cortical oncocytoma. There were no atypical mitotic figures, no venous invasion and no coagulative necrosis. Mitotic count was low, Ki67 was 3\% (low) and IGF2 was negative. Based on Lin-Weiss-Bisceglia classification, the tumour did not meet any major nor minor criteria and the tumour was classified as a benign oncocytoma. Despite these pathological findings, clinical and biochemical features of hyperandrogenism were considered adverse features; hence, long-term follow-up was planned.

\section{Outcome and follow-up}

Amenorrhoea, acne and hirsutism resolved rapidly post-operatively. Glucocorticoid cover was withdrawn and short Synachten test was normal. There was rapid improvement in her androgen levels following surgery. Results are summarised in Fig. 2. Her urinary steroid metabolite profile also improved. Results are summarised in Fig. 3 and Table 2.

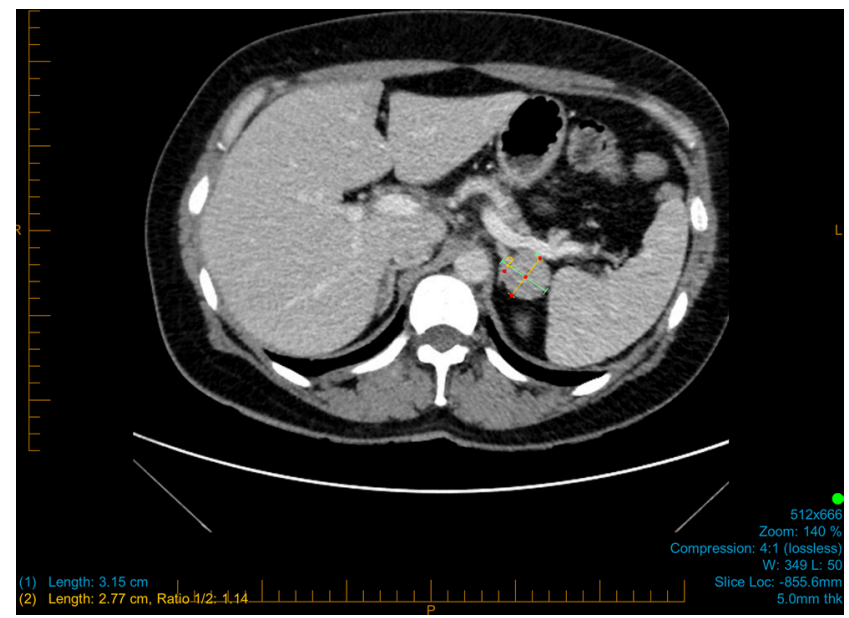

Figure 1

CT adrenals demonstrating left adrenal tumour. 
Table 2 24-h urine steroid metabolite data. Abnormal values are in bold face.

\section{Test}

Urine volume

Creatinine

Androsterone

Etiocholanolone

$5 \beta 17 \alpha$ hydroxypregnanolone

Pregnanetriol

Tetrahydro-11 deoxycortisol

Pregnanetriolone

Tetrahydro cortisone

Tetrahydro cortisol

\begin{tabular}{c}
\hline Pre-operatively \\
\hline 4.4 \\
3.59 \\
42.2 \\
45.8 \\
7.2 \\
20.1 \\
68.7 \\
$<0.5$ \\
14.2 \\
4.8
\end{tabular}

\begin{tabular}{c}
\hline Post-operatively \\
\hline 3.511 \\
5.53 \\
$\mathbf{2}$ \\
$\mathbf{1 . 5}$ \\
$\mathbf{1 . 1}$ \\
$\mathbf{1 . 7}$ \\
$\mathbf{0 . 2}$ \\
$<0.1$ \\
$\mathbf{7 . 8}$ \\
3.2 \\
\hline
\end{tabular}

\section{Discussion}

Adrenal masses are a common finding on cross-sectional abdominal imaging. Prevalence is $2 \%$ in the general population, with adrenocortical carcinoma (ACC) accounting for $2-11 \%$ of incidentally detected tumours (3, 4). Differentiating between tumour types is challenging. Serum biochemistry determines clinical functionality. Computed tomography (CT), magnetic resonance imaging (MRI) with chemical shift and ${ }^{18} \mathrm{~F}-2$-deoxy-D-glucose PET (FDG-PET/CT) are the most commonly used imaging techniques for differentiating malignant from benign adrenal tumours. However, data are sparse with limited number of small studies evaluating the sensitivity and specificity of each test $(3,4)$. Consensus guidelines suggest that tumours $\leq 4 \mathrm{~cm}$ with benign imaging characteristics do not require surgery. Conversely, tumours $>4 \mathrm{~cm}$ may require surgery, irrespective of imaging characteristics (4). Baseline CT density of $>10 \mathrm{HU}$ has a sensitivity of $100 \%$ and specificity of $72 \%$ for identifying malignant adrenal tumours $(3,4)$. Density $>10 \mathrm{HU}$ is also seen in benign lipid-poor adenomas and phaeochromocytomas. Other test performance estimates are based on very small numbers, with resulting wide $95 \%$ confidence intervals

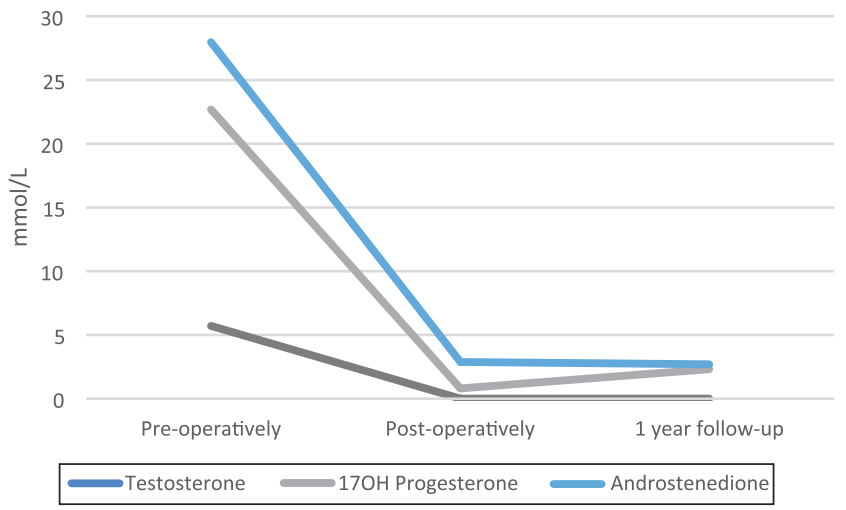

Figure 2

Resolution of serum hyperandrogenism post-operatively. and hence uncertainty in value. In this context, further diagnostic modalities and data are required for improved evaluation of adrenal tumours.

Urinary steroid metabolites have high sensitivity and specificity in differentiating of ACC from adrenocortical adenoma (ACA) $(1,3,5,6,7)$. ACC tumour cells are often dedifferentiated, and hence, produce precursors rather than end-products of steroidogenesis. The first large study to examine urine steroid metabolome in adrenocortical tumour patients was published by Arlt et al. in 2011 (3). Urinary steroids were examined by gas chromatographymass spectrometry (GC-MS) in 147 patients, of whom 45 had ACC. Hormonal overproduction was reported in 33/45 (73\%) of ACC patients. They demonstrated a 'malignant steroid fingerprint' consisting of metabolites that were most informative in differentiating malignant from nonmalignant tumours: androgen metabolite etiocholanolone (ET); androgen precursor metabolites pregnenediol (5-PD) and pregnenetriol (5-PT); mineralocorticoid metabolite 5-alpha-tetrahydro-11dehydro-corticosterone (5-alphaTHA); mineralocorticoid precursor metabolites tetrahydro11-deoxycorticosterone (THDOC); glucocorticoid precursor metabolites pregnanediol $(\mathrm{PD})$, pregnenatriol

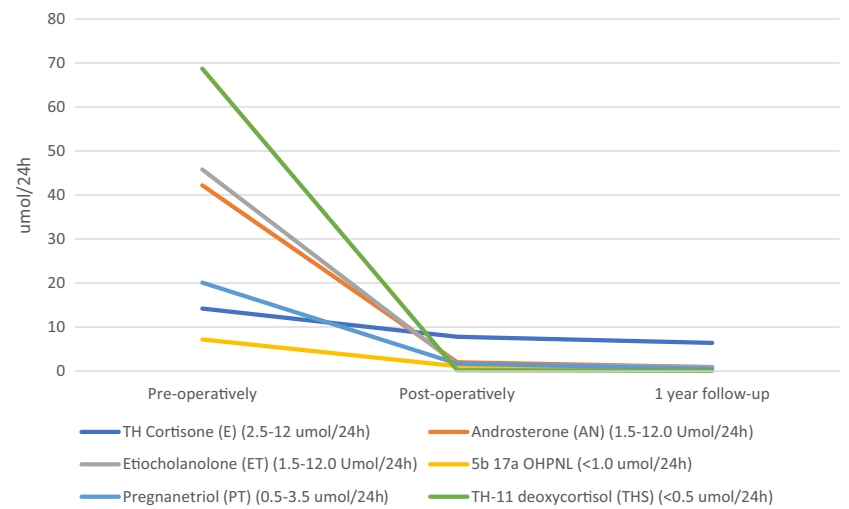

Figure 3

Resolution of urinary metabolites post-operatively. 
(PT) and THS and glucocorticoid metabolite 5-alphatetrahydrocortisol (5alpha-THF). The examination of the above metabolites yielded sensitivity and specificity of $90 \%$ on receiver-operating characteristic curve analysis. Of these, the three most important in identifying malignant tumours were 5-PT, 5-PD and THS (3). In a subsequent retrospective study, Kerkhofs et al. utilised GC-MS to examine the urinary steroid profile of 152 patients with adrenal tumours, of which 27 were ACC patients. Hormonal overproduction occurred in 20/27 ACC patients (74\%). They demonstrated higher concentration of 18 steroid metabolites in patients with ACC compared with those with other adrenal tumours. In particular, THS concentration of $>2.35 \mu \mathrm{mol} / 24 \mathrm{~h}$ differentiated ACC from other tumours with $100 \%$ sensitivity and $99 \%$ specificity (7). Other metabolites with $100 \%$ sensitivity for ACC were PD; PT; Etio; androsterone (An); tetrahydrocortisol and tetrahydrocortisone. However, specificities varied very widely (2-83\%). In 2016, Velikanova et al. used GC-MS to examine the urine metabolites of 139 patients with adrenocortical masses and 25 healthy persons. ACC diagnosis was histologically confirmed in 31 patients, of which 13 (42\%) had hormonal excess. Similarly, this study identified THS as the most significant, amongst other markers, in differentiating benign from malignant tumours. However, THS elevation was reported in only $74 \%$ of patients with ACC, and the authors suggested consideration of other metabolites and calculated ratios in order to increase sensitivity and specificity of the test (5). Within all three studies, the ACC metabolome was compared with that of other adrenal abnormalities, including ACA, benign myelolipoma, hyperplasia, multinodular hyperplasia, cavernous lymphangioma, ganglioneuroma, hemangioma, non-Hodgkin lymphoma and leiomyosarcoma. There were no cases of adrenal oncocytoma examined. This non-invasive test is a potentially useful adjunct to serum biochemistry and imaging commonly utilised for diagnosis of adrenal masses. One limitation will be availability of laboratories able to perform GC-MS. Higher throughput techniques will help to alleviate this problem.

Adrenal cortical oncocytomas are rare tumours, with approximately 160 cases reported in English literature. Most are incidentally detected, with only $17 \%$ being functional (8). Diagnosis is challenging, with no characteristic imaging features to differentiate from ACA and ACC. They commonly present as large tumours with varying degree of scattered lipid (8). Although fine-needle aspiration cytology has been proposed, this is contraindicated where malignancy is suspected. Given difficulties in identifying malignant tumours, this should not be performed (8). Tumours are characterised by large cells with dense eosinophilic cytoplasm, isolated nuclear pleomorphism and prominent nucleoli (9). The Lin-Weiss-Bisceglia classification differentiates between adrenal oncocytoma and adrenal oncocytic carcinoma (10). Presence of any of three major criteria indicates malignancy (high mitotic rate, atypical mitotic figures, venous invasion). Presence of any of four minor criteria (necrosis, sinusoidal invasion, capsular invasion, large size $>10 \mathrm{~cm}$ and/or weight $>200 \mathrm{~g}$ ) indicates uncertain malignant potential (10). The largest series of reported cases was published by Mearini et al. in 2014, with 147 identified cases from the literature (8). Tumours were more commonly reported in female patients and within the left adrenal. There were no identified risk factors (8). CT and MRI techniques are non-specific for adrenal oncocytoma and are not able to differentiate between benign and malignant oncocytic tumours. There are very few published cases of 18FDG-PET/CT in adrenal oncocytic tumours, with very mixed reports of efficacy (8). Adrenalectomy is the mainstay of therapy. Prognosis and long-term follow-up are not widely reported. Although careful follow-up is recommended, the modality for doing this remains unclear (8).

In summary, adrenal oncocytic tumours are rare neoplasms that pose a diagnostic and management challenge. Imaging studies have significant limitations in evaluation of adrenal masses, and in particular, oncocytic tumours. Urine steroid profiles have been utilised with promising success to identify malignant adrenal lesions. Herein, we report the first case of adrenal oncocytic tumour with evaluation of urinary steroid profile. Serum biochemistry confirmed clinical androgen excess. Imaging characteristics were consistent with a large, lipid-poor mass, raising concern for malignancy. Urinary steroid profile demonstrated elevation of several metabolites, in particular THS, which has a high sensitivity and specificity for malignancy. Urine steroid profile normalised following surgery, remaining within normal limits at 1-year follow-up. We propose that urinary steroid profile may be useful in diagnostic evaluation, prognostication and follow-up of adrenal tumours. Further research will assist in determining utility of urinary steroid profiles in non-functional adrenal tumours and for differentiating between benign and malignant oncocytic tumours. 


\section{Patient's perspective}

Initially I did not feel any different, but it was the unusual symptoms that were appearing that alerted me to something not being right. At no point would I have thought I was unwell - I did think that I was going through normal hormone changes due to my age.

The negative skins changes, excess unusual hair growth and that my periods did not return did raise concern. I was also noting that I was overly tired and that I could notice changes in my voice. This prompted me to attend my GP to see what was going on.

The initial tests I did were extensive blood tests of all hormones and an ultra sound as my GP thought I may have polycystic ovaries - this detected the adrenal issues.

The further tests I had to undergo were not in any way invasive, maybe just needed planning to complete the $24 \mathrm{~h}$ urine tests. I had many blood tests and adrenal function tests, none of which were painful or caused any side effects.

I was always prepared to have to undergo surgery and was happy to have this removed as what it was producing was far worse than any surgery. It did not take long to establish that surgery was needed and it was scheduled quickly. The surgery was fine, but it was very painful postsurgery as I was expecting. The recovery took a couple of weeks, but I do wish I took a little longer off work to fully rest post-surgery.

Since the surgery the adverse symptoms stopped almost immediately. The facial hair growth stopped; my skin cleared and my period returned within a month and has been regular ever since. My energy levels have also returned to normal. I understand that I may require more monitoring to ensure nothing else develops, which I am prepared to do whatever is required.

\section{Declaration of interest}

The authors declare that there is no conflict of interest that could be perceived as prejudicing the impartiality of this case report.

\section{Funding}

This research did not receive any specific grant from any funding agency in the public, commercial or not-for-profit sector.

\section{Patient consent}

Written consent form was obtained from the patient for publication of this article.

\section{Author contribution statement}

N L: literature review and drafting of manuscript; J G: primary physician, review of manuscript.

\section{References}

1 Bancos I \& Arlt W. Diagnosis of a malignant adrenal mass: the role of urinary steroid metabolite profiling. Current Opinion in Endocrinology, Diabetes, and Obesity 201724 200-207. (https://doi.org/10.1097/ MED.0000000000000333)

2 Panizzo V, Rubino B, Piozzi GN, Ubiali P, Morandi A, Nencioni M \& Micheletto G. Laparoscopic trans-abdominal right adrenalectomy for a large primitive adrenal oncocytic carcinoma: a case report and review of literature. American Journal of Case Reports 201819 1096-1102. (https://doi.org/10.12659/AJCR.910259)

3 Arlt W, Biehl M, Taylor AE, Hahner S, Libe R, Hughes BA, Schneider P, Smith DJ, Stiekema H, Krone N, et al. Urine steroid metabolomics as a biomarker tool for detecting malignancy in adrenal tumors. Journal of Clinical Endocrinology and Metabolism 2011 96 3775-3784. (https://doi.org/10.1210/jc.2011-1565)

4 Fassnacht M, Arlt W, Bancos I, Dralle H, Newell-Price J, Sahdev A, Tabarin A, Terzolo M, Tsagarakis S \& Dekkers OM. Management of adrenal incidentalomas: European Society of Endocrinology Clinical Practice Guideline in collaboration with the European Network for the Study of Adrenal Tumors. European Journal of Endocrinology 2016 175 G1-G34. (https://doi.org/10.1530/EJE-16-0467)

5 Velikanova LI, Shafigullina ZR, Lisitsin AA, Vorokhobina NV, Grigoryan K, Kukhianidze EA, Strelnikova EG, Krivokhizhina NS, Krasnov LM, Fedorov EA, et al. Different types of urinary steroid profiling obtained by high-performance liquid chromatography and gas chromatography-mass spectrometry in patients with adrenocortical carcinoma. Hormones and Cancer 20167 327-335. (https://doi.org/10.1007/s12672-016-0267-0)

6 Shafigullina ZR, Velikanova LI, Vorokhobina NV, Shustov SB, Lisitsin AA, Malevanaia EV, Buinova MO, Bessonova EA \& Kirsanov DO. Urinary steroid profiling by gas chromatography mass spectrometry: early features of malignancy in patients with adrenal incidentalomas. Steroids 2018135 31-35. (https://doi.org/10.1016/j. steroids.2018.04.006)

7 Kerkhofs TM, Kerstens MN, Kema IP, Willems TP \& Haak HR. Diagnostic value of urinary steroid profiling in the evaluation of adrenal tumors. Hormones and Cancer 20156 168-175. (https://doi. org/10.1007/s12672-015-0224-3)

8 Mearini L, Del Sordo R, Costantini E, Nunzi E \& Porena M. Adrenal oncocytic neoplasm: a systematic review. Urologia Internationalis 2013 91 125-133. (https://doi.org/10.1159/000345141)

9 Lam AK. Update on adrenal tumours in 2017 World Health Organization (WHO) of endocrine tumours. Endocrine Pathology 2017 28 213-227. (https://doi.org/10.1007/s12022-017-9484-5)

10 Bisceglia M, Ludovico O, Di Mattia A, Ben-Dor D, Sandbank J, Pasquinelli G, Lau SK \& Weiss LM. Adrenocortical oncocytic tumors: report of 10 cases and review of the literature. International Journal of Surgical Pathology 200412 231-243. (https://doi. org/10.1177/106689690401200304)

Received in final form 23 September 2019 Accepted 6 November 2019 